\title{
Tuning parametric models of the atmospheric muon flux in MUPAGE to data from the KM3NeT detector
}

\section{Brían Ó Fearraigh ${ }^{a, b, *}$ on behalf of the KM3NeT Collaboration}

(a complete list of authors can be found at the end of the proceedings)

${ }^{a}$ Nikhef, National Institute for Subatomic Physics,

PO Box 41882, Amsterdam, 1009 DB The Netherlands

${ }^{b}$ University of Amsterdam,

Institute of Physics/IHEF, PO Box 94216, Amsterdam, 1090 GE Netherlands

E-mail: ofearrab@nikhef.nl

The muons produced by cosmic ray interactions in the upper atmosphere constitute the most abundant signal for underwater neutrino detectors such as KM3NeT (the Cubic Kilometre Neutrino Telescope), which is currently being deployed in the Mediterranean Sea at two distinct locations. Situated at different depths, the KM3NeT/ARCA and KM3NeT/ORCA detectors experience a different flux of muons, and thus are uniquely positioned to study their evolution and propagation from cosmic ray showers. It is imperative to the main physics goals of the experiment that the atmospheric muon background is modelled correctly, which aids in benchmarking and understanding the detector response to the constant flux of these particles.

In this study, the data from the KM3NeT/ORCA detector is used and compared with the Monte Carlo (MC) prediction from the MUPAGE (MUons from PArametric formulas: a fast GEnerator for neutrino telescopes) software package, which generates the energy spectrum, lateral distribution, and muon multiplicity of muon bundles according to a specific parametrisation at different depths below sea level. This parametrisation consists of many free parameters which can be tuned such that simulated physical observables in the detector agree with those measured in data. In this way, improvements to the data-MC agreement are achieved by quantitatively comparing the level of agreement between simulated and measured observables in the KM3NeT detector.

$37^{\text {th }}$ International Cosmic Ray Conference (ICRC 2021)

July 12th - 23rd, 2021

Online - Berlin, Germany

\footnotetext{
${ }^{*}$ Presenter
} 


\section{Contents}

1 Introduction 2

2 The KM3NeT Detector 3

3 Simulation Chain 3

4 Monte Carlo Comparisons 4

5 Comparison Methods $\quad 4$

6 Data-MC Comparisons 5

7 Discussion 6

\section{Introduction}

Atmospheric muons constitute the most abundant signal for many neutrino experiments; they require careful treatment in experimental data in order to disentangle them from neutrino events. These muons however, created in cosmic ray interactions in the upper atmosphere, offer in themselves a rich study of cosmic ray physics.

There are outstanding uncertainties in the modelling and understanding of cosmic ray-induced particle showers, the most prominent being the energy-dependent primary composition and the different hadronic models under consideration, with uncertainties which span vast energy ranges $[1,2]$. These uncertainties in turn affect the atmospheric neutrino flux models used in atmospheric neutrino physics analyses [3].

The KM3NeT underwater neutrino detectors [4] offer an additional probe to these cosmic ray model uncertainties. With nanosecond-level timing resolution, KM3NeT has unprecedented access to high-energy atmospheric muons which reach the depths of Mediterranean Sea, and can probe the transverse momentum of these particles. Through the distinguishing of individual high-energy muons, KM3NeT can contribute to an understanding of the early-stage cosmic ray interactions.

Thus, the atmospheric muon simulation in KM3NeT needs to describe the data to a high-degree of accuracy. MUPAGE [5, 6], a fast Monte Carlo generator of atmospheric muon bundles for underwater or in-ice neutrino detectors, generates muon bundles according to a specific parametrisation. Its internal parametric formulae consists of many free parameters, which can be varied. The methodology behind tuning the parameters in MUPAGE such that simulation better agrees with $\mathrm{KM} 3 \mathrm{NeT}$ data is described in this contribution. 


\section{The KM3NeT Detector}

$\mathrm{KM} 3 \mathrm{NeT}$ is a network of neutrino detectors under construction at two locations in the Mediterranean Sea. Each detector comprises a volume of sea water where an array of photo-sensors detects the Cherenkov radiation [8] induced by the charged particles created in neutrino interactions.

The detection modules, or 'optical modules', comprise a pressure-resistant glass sphere which houses 31 3-inch PMTs alongside a Central Logic Board and accompanying components and electronics [4, 7]. A 'detection unit' is formed by 18 optical modules arranged along a vertical line, with a full detector 'building block' consisting of 115 detection units. Different distances between the optical modules along the detection units and between detection units within the building blocks themselves allow for KM3NeT to achieve different physics goals.

The KM3NeT/ARCA detector primarily focuses on neutrino astronomy, with the objective to identify sources of cosmic neutrinos. With an average spacing between optical modules of $36 \mathrm{~m}$, and $90 \mathrm{~m}$ between detection units, KM3NeT/ARCA is optimised to detect neutrinos in the TeV-PeV energy range. Comprising two building blocks upon completion, KM3NeT/ARCA will enclose $2 \mathrm{x}$ $0.48 \mathrm{~km}^{3}$ of sea water. It is located at $36^{\circ} 16^{\prime} \mathrm{N} 16^{\circ} 06^{\prime} \mathrm{E}$ at a depth of $3.5 \mathrm{~km}, 100 \mathrm{~km}$ offshore of Porto Palo di Capo Passero, Sicily.

KM3NeT/ORCA is a dense configuration of the building block, with an average spacing between optical modules of $9 \mathrm{~m}$ along a detection unit, and $20 \mathrm{~m}$ between detection units themselves. This geometry is optimised for the detection of neutrinos in the few $\mathrm{GeV}$ to $100 \mathrm{GeV}$ range. KM3NeT/ORCA aims to study neutrino oscillations, constrain the oscillation parameters and determine the yet-unknown neutrino mass ordering. The infrastructure for the detector is located at $42^{\circ} 48^{\prime} \mathrm{N} 06^{\circ} 02^{\prime} \mathrm{E}$ at a depth of $2.45 \mathrm{~km}$, about $40 \mathrm{~km}$ offshore from Toulon, France.

At the time of writing, KM3NeT/ARCA and KM3NeT/ORCA are operating with 6 detection units each, continuously taking data in the Mediterranean Sea. A data run from the ORCA-6 partdetector is chosen for the purposes of this study, namely run number 7398, due to its high quality. It is this data run which is simulated in this research study and against which different MUPAGE parameters are compared.

\section{Simulation Chain}

The series of software programs used to carry out a simulation will be referred to as the 'simulation chain'. For the purposes of this study, the data run must be simulated as realistically as possible, attempting to replicate the exact data-taking conditions of the detector during the specific time period.

The simulation chain follows four stages.

1. The generator stage involves the generation of particles, namely atmospheric muons. MUPAGE provides a fast method of generating muons on the surface of a virtual cylinder - which encloses the detector - according to a specific parameterisation. Within MUPAGE there are numerous free parameters which describe the underlying generated distributions of the muon flux, lateral spread (radial distance of muons from primary shower axis), single muon energy spectrum and multiple muon energy spectrum. The muons reach the detector volume and the rest of the simulation chain is carried out. 
2. The light stage simulates the emission of Cherenkov radiation and its detection.

3. The trigger stage simulates the PMT and detector response, as well as trigger settings (the specific detector conditions used to filter data).

4. Finally, in the reconstruction stage, the trajectory of the simulated muons are determined by confronting the time-of-arrival of light with the expected arrival time. A log-likelihood fit can be used to estimate the direction and energy of the muons. Following the reconstruction stage, distributions of physical observables can be produced. These include the reconstructed direction and the reconstructed energy.

In this contribution, the approach towards tuning the parameters within MUPAGE to better agree with data is outlined. In essence, the simulation chain is carried out for different values of the MUPAGE parameters, and the reconstructed observables are compared between simulation and data. The current values for all of these parameters, as defined in [6], are referred to as the nominal values.

\section{Monte Carlo Comparisons}

The parameters which most affect the distributions of observables are identified. This is achieved by simulating the ORCA- 6 run 7398 and varying the individual MUPAGE parameters by the same amount, keeping the rest fixed. The simulation chain steps of light, trigger and reconstruction are kept the same for every simulation of varied MUPAGE parameters. An example of this comparison between simulation only is shown in Figure 1. $\beta$, a parameter that describes the energy loss in the parametric equations of MUPAGE [6], is shown to have an effect on the shape of the reconstructed direction when it is varied. The fact that a parameter which describes the energy can affect the direction shows the complexity of the exercise. From this comparison between simulations, six parameters are identified with noticeable impacts on the observables: $\beta$, $v_{1 b}, K_{1 a}, d_{0 b}, b_{1 b}$ and $\rho_{0 b}$.

\section{Comparison Methods}

In order to compare distributions of data and simulation, a quantitative measurement of the level of agreement between distributions - obtained with different values of the MUPAGE parameters - is required. One such metric, measuring the difference between distributions, is the significance [9].

When comparing two histograms $H_{a}$ and $H_{b}$, the significance $S$ can provide a measure of the difference between them. It can be defined as:

$$
S=\frac{1}{N} \sum_{i=0}^{N} \frac{\left|a_{i}-K \cdot b_{i}\right|}{\sigma^{2}\left(a_{i}+K^{2} \cdot \sigma^{2}\left(b_{i}\right)\right)},
$$

where $N$ is the number of entries in $H_{a}$ and $H_{b}, a_{i}$ and $b_{i}$ are the contents of bin $i$ in both histograms, $K$ is the scaling parameter between the two histograms, and the error $\sigma$ assumes the contents follow Poisson distributions. It can be shown that when $H_{a}=H_{b}, S=0$. 


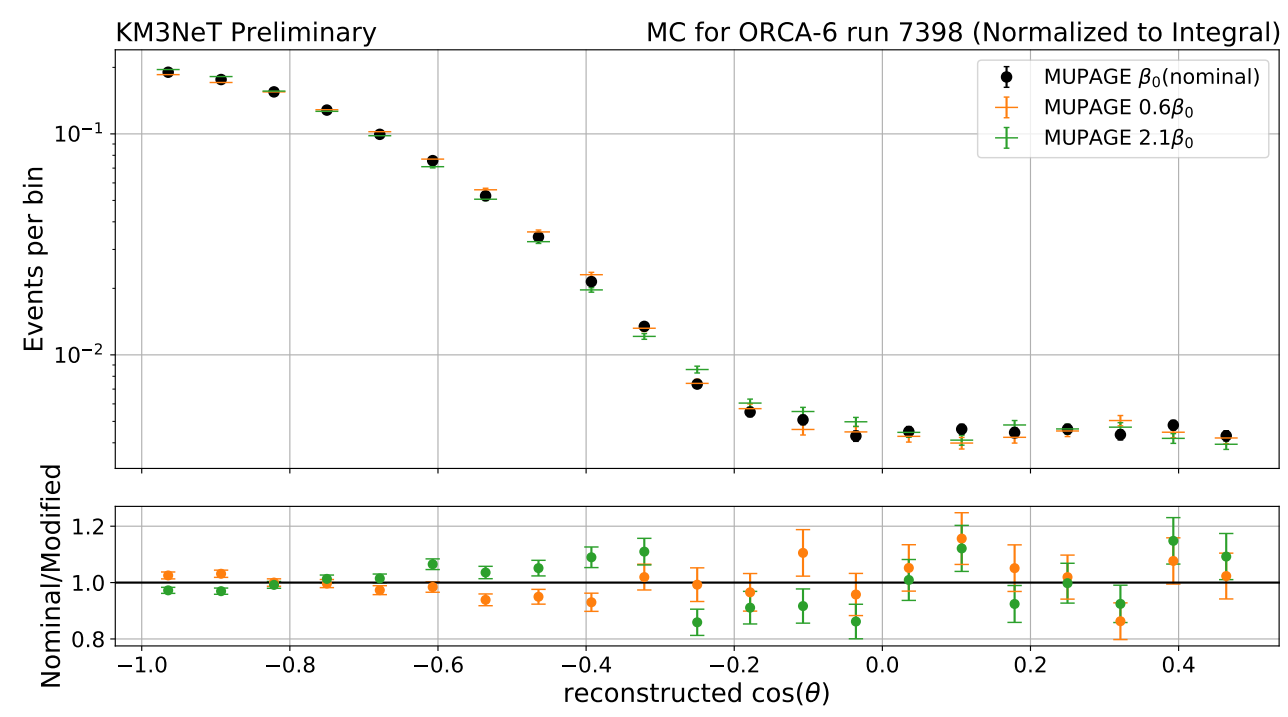

Figure 1: Reconstructed direction $\cos (\theta)$ for different values of MUPAGE parameter $\beta$, simulating the ORCA-6 data run 7398. The top figure has been normalized to show the differences in shape only. The bottom figure shows the nominal:varied MUPAGE parameters ratio for simulation of $\beta=0.6 \beta_{0}$ (orange points) compared to $\beta=2.1 \beta_{0}$ (green points).

Thus, in the context of tuning MUPAGE parameters to improve the agreement with data, a value of $S$ approaching 0 is desired. The value of the significance between observables in data and simulation for the nominal MUPAGE parameters provides the benchmark to improve upon. For this contribution, the agreement in the shape of the distributions is in focus.

\section{Data-MC Comparisons}

The following results are from a preliminary scan of the MUPAGE parameter phase space. Values of $\beta, v_{1 b}, K_{1 a}, d_{0 b}, b_{1 b}$ and $\rho_{0 b}$ are varied one at a time, i.e. independently from another, across a range of parameter values (namely, 0.6 - 2.1 times the nominal value). The ORCA- 6 data run 7398 is thus simulated with these varying parameters. The observables in data and simulation are then compared to one another using the significance test. Note that simple cuts of $\cos \theta<0.5$ and reconstructed $E>5 \mathrm{GeV}$ are applied to remove regions of noise.

From this, candidate parameter values giving a better data-MC agreement than the nominal MUPAGE parameters are found. For each simulation run, with a unique set of MUPAGE parameters, a significance score for the reconstructed direction, reconstructed energy, number of triggered hits, number of triggers per event and reconstruction quality is obtained when simulated observables are compared to the same observables in data. An average value of the significance is then calculated to associate one significance value to a particular simulation chain run.

For reference, the average significance of the comparison between data and a MC simulation with the nominal MUPAGE parameter values is

$$
S_{\text {avg,nominal }}=2.418
$$


A particular simulation is found which gives the lowest $S_{a v g}$ across all parameter combinations. The reconstructed $\cos \theta$ distribution of this simulation is shown in Figure 2 for data, the nominal MUPAGE parameter simulation and the best-agreement set of parameter values. In this figure the influence of the normalization is removed. This new parameter set clearly results in a better data-MC agreement than for the nominal MUPAGE case. It serves as a proof of this method for parameter tuning.

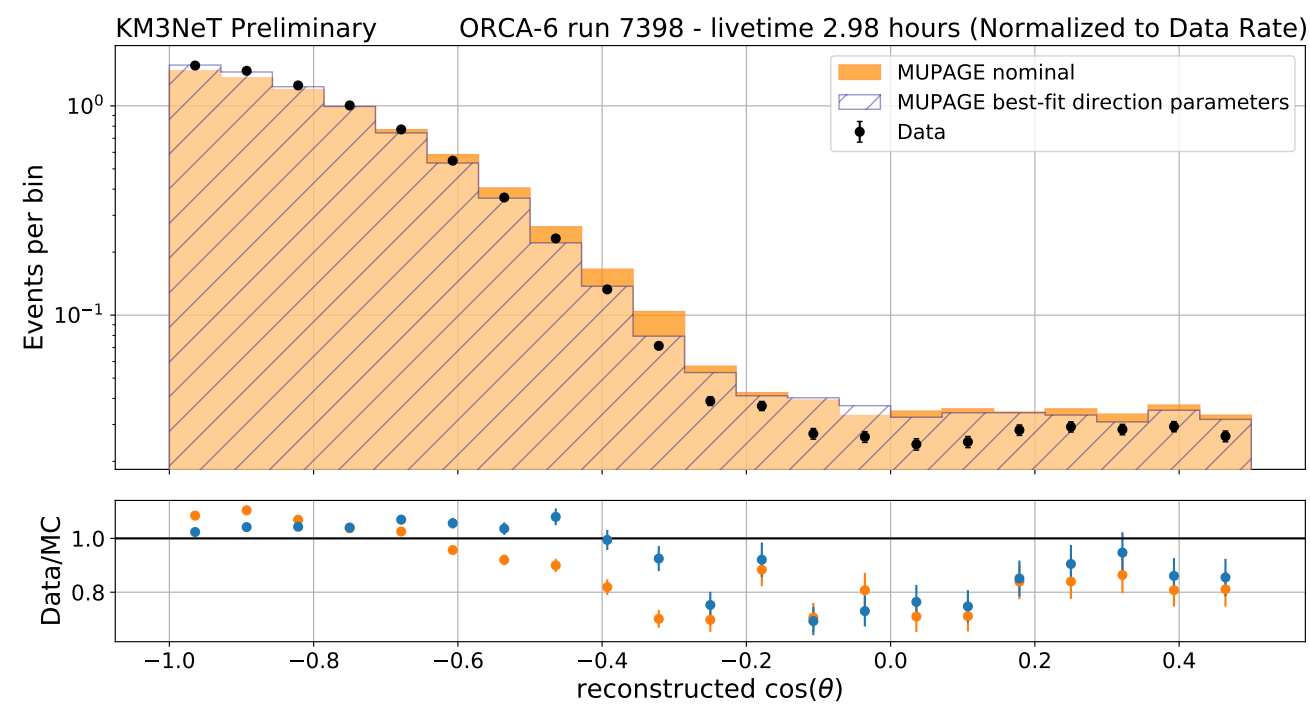

Figure 2: Reconstructed direction $\cos (\theta)$ for data, nominal MUPAGE parameters and a best-agreement simulation. The top figure has been normalized to remove the effects of direct scaling. The bottom figure shows the data:MC ratio for nominal MUPAGE parameters (orange points) compared to the agreement for the best-fit direction parameters (blue points).

With a multitude of observables being compared, the minimum $S_{a v g}$ is not necessarily the parameter set giving the best agreement amongst all the observables. Another distribution, namely the reconstructed energy, is shown in Figure 3. Here a value of $S<S_{\text {avg,nominal }}$ is found for a different parameter set. As can be seen, a slight improvement for this new parameter set is found compared to the nominal MUPAGE case. Again this conveys the complexity of the exercise, and illustrates that the shape can be varied.

\section{Discussion}

A preliminary scan of the parameter space has been carried out to tune MUPAGE parameters to KM3NeT data, in order to improve the description of the data by the MC. The method of comparing simulated and data observables with the significance test is validated, illustrating that varied MUPAGE parameters with certain significance values can improve the data-MC agreement with respect to that of the nominal MUPAGE parameters. A tunable parametric simulation can be carried out, paving the way towards high-precision measurements of physics observables by KM3NeT, with a MC simulation which describes the data to a high-degree of accuracy. A multi- 

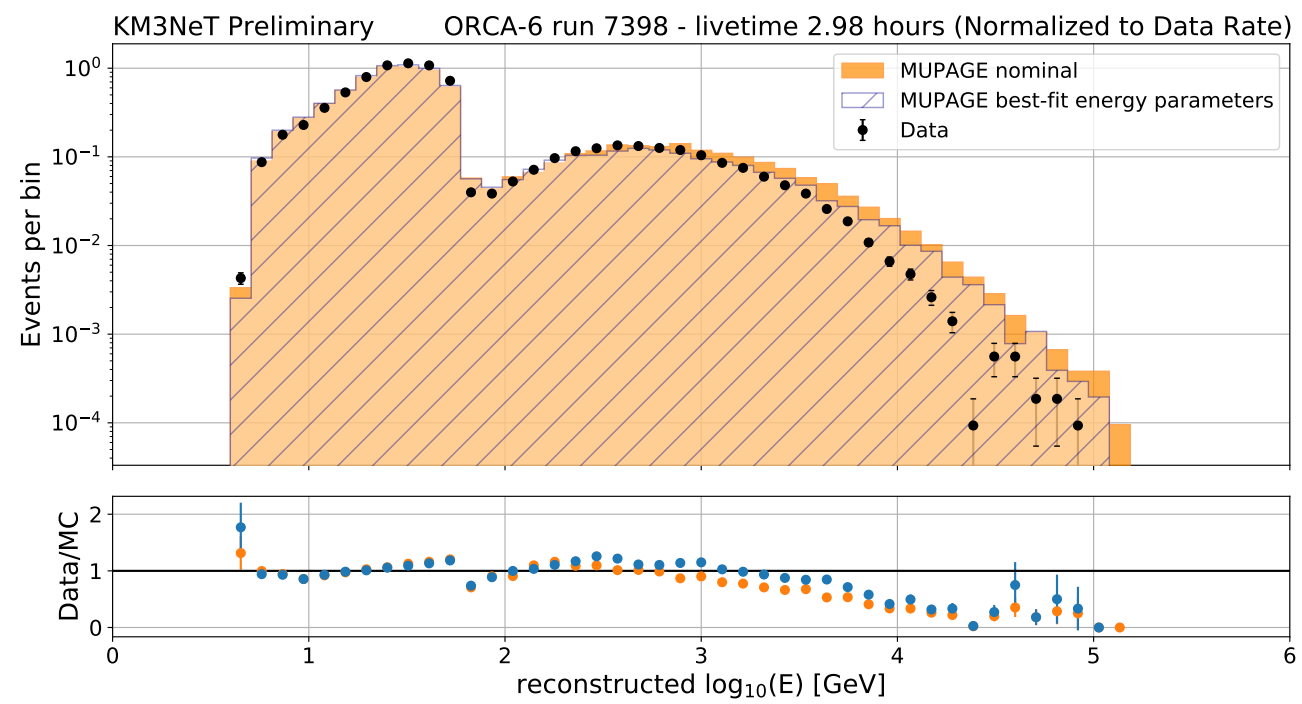

Figure 3: Reconstructed energy for data, nominal MUPAGE parameters and a best-agreement simulation. The top figure has been normalized to remove the effects of direct scaling. The bottom figure shows the data:MC ratio for nominal MUPAGE parameters (orange points) compared to the agreement for the best-fit energy parameters (blue points).

dimensional scan of the parameter space is intended to be carried out as a follow-up to this work in order to identify the MUPAGE parameter values which provide further agreement with data. 


\section{References}

[1] R. D. Parsons and H. Schoorlemmer, "Systematic Differences due to High Energy Hadronic Interaction Models in Air Shower Simulations in the $100 \mathrm{GeV}-100 \mathrm{TeV}$ Range" Phys. Rev. D 100 (2019), 023010 doi:10.1103/PhysRevD.100.023010 [arXiv:1904.05135].

[2] A. Fedynitch, F. Riehn, R. Engel, T. K. Gaisser and T. Stanev, "Hadronic interaction model sibyll 2.3c and inclusive lepton fluxes" Phys. Rev. D 100 (2019), 103018 doi:10.1103/PhysRevD.100.103018 [arXiv:1806.04140].

[3] J. Evans, D. G. Gamez, S. D. Porzio, S. Söldner-Rembold and S. Wren, "Uncertainties in Atmospheric Muon-Neutrino Fluxes Arising from Cosmic-Ray Primaries" Phys. Rev. D 95 (2017), 023012 doi:10.1103/PhysRevD.95.023012 [arXiv:1612.03219].

[4] S. Adrian-Martinez et al. [KM3Net], "Letter of intent for KM3NeT 2.0” J. Phys. G 43 (2016), 084001 doi:10.1088/0954-3899/43/8/084001 [arXiv:1601.07459].

[5] G. Carminati, A. Margiotta and M. Spurio, "Atmospheric MUons from PArametric formulas: A Fast GEnerator for neutrino telescopes (MUPAGE)" Comput. Phys. Commun. 179 (2008), 915 doi:10.1016/j.cpc.2008.07.014 [arXiv:0802.0562].

[6] Y. Becherini, A. Margiotta, M. Sioli and M. Spurio, "A Parameterisation of single and multiple muons in the deep water or ice" Astropart. Phys. 25 (2006), 1 doi:10.1016/j.astropartphys.2005.10.005 [arXiv:hep-ph/0507228].

[7] S. Aiello et al. [KM3NeT], "KM3NeT front-end and readout electronics system: hardware, firmware and software", J. Astron. Telesc. Instrum. Syst. 5 (2019) 046001 doi:10.1117/1.JATIS.5.4.046001 [arXiv:1907.06453].

[8] P. A. Cerenkov, "Visible radiation produced by electrons moving in a medium with velocities exceeding that of light" Phys. Rev. 52 (1937), 378 doi:10.1103/PhysRev.52.378.

[9] T. P. Li and Y. Q. Ma, "Analysis methods for results in gamma-ray astronomy” Astrophys. J. 272 (1983), 317-324 doi:10.1086/161295. 


\section{Full Authors List: KM3NeT Collaboration}

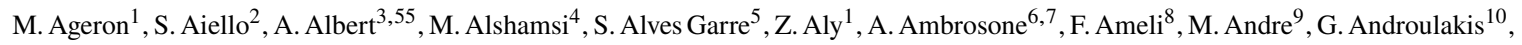
M. Anghinolfi1 11 , M. Anguita ${ }^{12}$, G. Anton ${ }^{13}$, M. Ardid ${ }^{14}$, S. Ardid ${ }^{14}$, W. Assal ${ }^{1}$, J. Aublin ${ }^{4}$, C. Bagatelas ${ }^{10}$, B. Baret ${ }^{4}$, S. Basegmez du Pree ${ }^{15}$, M. Bendahman ${ }^{4,16}$, F. Benfenati ${ }^{17,18}$, E. Berbee ${ }^{15}$, A. M. van den Berg ${ }^{19}$, V. Bertin ${ }^{1}$, S. Beurthey ${ }^{1}$, V. van Beveren ${ }^{15}$, S. Biagi ${ }^{20}$, M. Billault ${ }^{1}$, M. Bissinger ${ }^{13}$, M. Boettcher ${ }^{21}$, M. Bou Cabo ${ }^{22}$, J. Boumaaza ${ }^{6}$, M. Bouta ${ }^{23}$, C. Boutonnet ${ }^{4}$, G. Bouvet ${ }^{24}$, M. Bouwhuis ${ }^{15}$, C. Bozza ${ }^{25}$, H.Brânzass ${ }^{26}$, R. Bruijn ${ }^{15,27}$, J. Brunner ${ }^{1}$, R. Bruno ${ }^{2}$, E. Buis ${ }^{28}$, R. Buompane ${ }^{6,29}$, J. Busto ${ }^{1}$, B. Caiffi ${ }^{11}$, L. Caillat ${ }^{1}$, D. Calvo ${ }^{5}$, S. Campion ${ }^{30,8}$, A. Capone ${ }^{30,8}$, H. Carduner ${ }^{24}$, V. Carretero ${ }^{5}$, P. Castaldi ${ }^{17,31}$, S. Celli ${ }^{30,8}$, R. Cereseto ${ }^{11}$, M. Chabab ${ }^{32}$, C. Champion ${ }^{4}$, N. $\mathrm{Chau}^{4}$, A. Chen ${ }^{33}$, S. Cherubinini ${ }^{20,34}$, V. Chiarella ${ }^{35}$, T. Chiarusi ${ }^{17}$, M. Circella ${ }^{36}$, R. Cocimano ${ }^{20}$, J. A. B. Coelho ${ }^{4}$, A. Coleiro ${ }^{4}$, M. Colomer Molla ${ }^{4,5}$, S. Colonges ${ }^{4}$, R. Coniglione ${ }^{20}$, A. Cosquer ${ }^{1}$, P. Coyle ${ }^{1}$, M. Cresta ${ }^{11}$, A. Creusot ${ }^{4}$, A. $\mathrm{Cruz}^{37}$, G. Cuttone ${ }^{20}$, A. D’Amico ${ }^{15}$, R. Dallier ${ }^{24}$, B. De Martino ${ }^{1}$, M. De Palma ${ }^{36,38}$, I. Di Palma ${ }^{30,8}$, A. F. Díaz ${ }^{12}$, D. Diego$\operatorname{Tortosa}^{14}$, C. Distefano ${ }^{20}$, A. Domi ${ }^{15,27}$, C. Donzaud ${ }^{4}$, D. Dornic ${ }^{1}$, M. Dörr ${ }^{39}$, D. Drouhin ${ }^{3,55}$, T. Eberl ${ }^{13}$, A. Eddyamoui ${ }^{16}$, T. van Eeden ${ }^{15}$, D. van Eijk ${ }^{15}$, I. El Bojaddaini ${ }^{23}$, H. Eljarrari ${ }^{16}$, D. Elsaesser ${ }^{39}$, A. Enzenhöfer ${ }^{1}$, V. Espinosa ${ }^{14}$, P. Fermani ${ }^{30,8}$, G. Ferrara ${ }^{20,34}$, M. D. Filipović ${ }^{40}$, F. Filippini ${ }^{17,18}$, J. Fransen ${ }^{15}$, L. A. Fusco ${ }^{1}$, D. Gajanana ${ }^{15}$, T. Gal ${ }^{13}$, J. García Méndez ${ }^{14}$, A. Garcia Soto $^{5}$, E. Garçon ${ }^{1}$, F. Garufi ${ }^{6,7}$, C. Gatius ${ }^{15}$, N. Geißelbrecht ${ }^{13}$, L. Gialanella ${ }^{6,29}$, E. Giorgio ${ }^{20}$, S. R. Gozzini ${ }^{5}$, R. Gracia ${ }^{15}$, K. Graf ${ }^{13}$, G. Grella ${ }^{41}$, D. Guderian ${ }^{56}$, C. Guidi ${ }^{11,42}$, B. Guillon ${ }^{43}$, M. Gutiérrez ${ }^{44}$, J. Haefner ${ }^{13}$, S. Hallmann ${ }^{13}$, H. Hamdaoui ${ }^{16}$, H. van Haren ${ }^{45}$, A. Heijboer ${ }^{15}$, A. Hekalo ${ }^{39}$, L. Hennig ${ }^{13}$, S. Henry ${ }^{1}$, J. J. Hernández-Rey ${ }^{5}$, J. Hofestädt ${ }^{13}$, F. Huang ${ }^{1}$, W. Idrissi Ibnsalih ${ }^{6,29}$, A. Ilioni ${ }^{4}$, G. Illuminati ${ }^{17,18,4}$, C. W. James ${ }^{37}$, D. Janezashvili ${ }^{46}$, P. Jansweijer ${ }^{15}$, M. de Jong ${ }^{15,47}$, P. de Jong ${ }^{15,27}$, B. J. Jung ${ }^{15}$, M. Kadler ${ }^{39}$, P. Kalaczyński ${ }^{48}$, O. Kalekin ${ }^{13}$, U. F. Katz ${ }^{13}$, F. Kayzel $^{15}$, P. Keller ${ }^{1}$, N. R. Khan Chowdhury ${ }^{5}$, G. Kistauri ${ }^{46}$, F. van der Knaap ${ }^{28}$, P. Kooijman ${ }^{27,57}$, A. Kouchner ${ }^{4,49}$, M. Kreter ${ }^{21}$, V. Kulikovskiy ${ }^{11}$, M. Labalme ${ }^{43}$, P. Lagier ${ }^{1}$, R. Lahmann ${ }^{13}$, P. Lamare ${ }^{1}$, M. Lamoureux $^{14}$, G. Larosa ${ }^{20}$, C. Lastoria ${ }^{1}$, J. Laurence ${ }^{1}$, A. Lazo $^{5}$, R. Le Breton ${ }^{4}$, E. Le Guirriec ${ }^{1}$, S. Le Stum ${ }^{1}$, G. Lehaut ${ }^{43}$, O. Leonardi ${ }^{20}$, F. Leone ${ }^{20,34}$, E. Leonora ${ }^{2}$, C. Lerouvillois ${ }^{1}$, J. Lesrel ${ }^{4}$, N. Lessing ${ }^{13}$, G. Levi ${ }^{17,18}$, M. Lincetto ${ }^{1}$, M. Lindsey Clark ${ }^{4}$, T. Lipreau ${ }^{24}$, C. LLorens Alvarez ${ }^{14}$, A. Lonardo ${ }^{8}$, F. Longhitano ${ }^{2}$, D. Lopez-Coto ${ }^{44}$, N. Lumb ${ }^{1}$, L. Maderer ${ }^{4}$, J. Majumdar ${ }^{15}$, J. Mańczak ${ }^{5}$, A. Margiotta ${ }^{17,18}$, A. Marinelli ${ }^{6}$, A. Marini ${ }^{1}$, C. Markou $^{10}$, L. Martin ${ }^{24}$, J. A. Martínez-Mora ${ }^{14}$, A. Martini ${ }^{35}$, F. Marzaioli ${ }^{6,29}$,

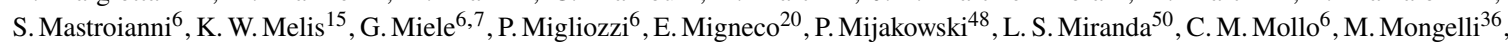
A. Moussa ${ }^{23}$, R. Muller ${ }^{15}$, P. Musico ${ }^{11}$, M. Musumeci ${ }^{20}$, L. Nauta ${ }^{15}$, S. Navas ${ }^{44}$, C. A. Nicolau ${ }^{8}$, B. Nkosi ${ }^{33}$, B. Ó Fearraigh ${ }^{15,27}$, M. O'Sullivan ${ }^{37}$, A. Orlando ${ }^{20}$, G. Ottonello ${ }^{11}$, S. Ottonello ${ }^{11}$, J. Palacios González ${ }^{5}$, G. Papalashviliite, R. Papaleo ${ }^{20}$, C. Pastore ${ }^{36}$, A. M. Păun ${ }^{26}$, G. E. Păvălaş ${ }^{26}$, G. Pellegrinini ${ }^{17}$, C. Pellegrino ${ }^{18,58}$, M. Perrin-Terrini ${ }^{1}$, V. Pestel ${ }^{15}$, P. Piattelli ${ }^{20}$, C. Pieterse ${ }^{5}$, O. Pisanti ${ }^{6,7}$, C. Poirè ${ }^{14}$, V. Popa $^{26}$, T. Pradier $^{3}$, F. Pratolongo ${ }^{11}$, I. Probst ${ }^{13}$, G. Pühlhofer ${ }^{51}$, S. Pulvirenti ${ }^{20}$, G. Quéméner ${ }^{43}$, N. Randazzo ${ }^{2}$, A. Rapicavoli ${ }^{34}$, S. Razzaque $^{50}$, D. Real ${ }^{5}$, S. Reck ${ }^{13}$, G. Riccobene ${ }^{20}$, L. Rigalleau ${ }^{24}$, A. Romanov ${ }^{11,42}$, A. Rovelli ${ }^{20}$, J. Royon ${ }^{1}$, F. Salesa Greus ${ }^{5}$, D. F. E. Samtleben ${ }^{15,47}$, A. Sánchez Losa ${ }^{36,5}$, M. Sanguineti ${ }^{11,42}$, A. Santangelo ${ }^{51}$, D. Santonocito ${ }^{20}$, P. Sapienza ${ }^{20}$, J. Schmelling ${ }^{15}$, J. Schnabel ${ }^{13}$, M. F. Schneider ${ }^{13}$, J. Schumann ${ }^{13}$, H. M. Schutte ${ }^{21}$, J. Seneca ${ }^{15}$, I. Sgura ${ }^{36}$, R. Shanidze ${ }^{46}$, A. Sharma ${ }^{52}$, A. Sinopoulou ${ }^{10}$, B. Spisso ${ }^{41,6}$, M. Spurio ${ }^{17,18}$, D. Stavropoulos ${ }^{10}$, J. Steijger ${ }^{15}$, S. M. Stellacci ${ }^{41,6}$, M. Taiuti ${ }^{11,42}$, F. Tatone ${ }^{36}$, Y. Tayalati ${ }^{16}$, E. Tenllado ${ }^{44}$, D. Tézier ${ }^{1}$, T. Thakore ${ }^{5}$, S. Theraube ${ }^{1}$, H. Thiersen $^{21}$, P. Timmer $^{15}$, S. Tingay ${ }^{37}$, S. Tsagkli $^{10}$, V. Tsourapis ${ }^{10}$, E. Tzamariudaki ${ }^{10}$, D. Tzanetatos ${ }^{10}$, C. Valieri ${ }^{17}$, V. Van Elewyck ${ }^{4,49}$, G. Vasileiadis ${ }^{53}$, F. Versari ${ }^{17,18}$, S. Viola $^{20}$, D. Vivolo ${ }^{6,29}$, G. de Wasseige ${ }^{4}$, J. Wilms ${ }^{54}$, R. Wojaczyński ${ }^{48}$, E. de Wolf ${ }^{15,27}$, T. Yousfi ${ }^{23}$, S. Zavatarelli ${ }^{11}$, A. Zegarelli ${ }^{30,8}$, D. Zito ${ }^{20}$, J. D. Zornoza ${ }^{5}$, J. Zúñiga ${ }^{5}$, N. Zywucka ${ }^{21}$.

${ }^{1}$ Aix Marseille Univ, CNRS/IN2P3, CPPM, Marseille, France.

${ }^{2}$ INFN, Sezione di Catania, Via Santa Sofia 64, Catania, 95123 Italy.

${ }^{3}$ Université de Strasbourg, CNRS, IPHC UMR 7178, F-67000 Strasbourg, France.

${ }^{4}$ Université de Paris, CNRS, Astroparticule et Cosmologie, F-75013 Paris, France.

${ }^{5}$ IFIC - Instituto de Física Corpuscular (CSIC - Universitat de València), c/Catedrático José Beltrán, 2, 46980 Paterna, Valencia, Spain. ${ }^{6}$ INFN, Sezione di Napoli, Complesso Universitario di Monte S. Angelo, Via Cintia ed. G, Napoli, 80126 Italy.

${ }^{7}$ Università di Napoli “Federico II”, Dip. Scienze Fisiche "E. Pancini”, Complesso Universitario di Monte S. Angelo, Via Cintia ed. G, Napoli, 80126 Italy.

${ }^{8}$ INFN, Sezione di Roma, Piazzale Aldo Moro 2, Roma, 00185 Italy.

${ }^{9}$ Universitat Politècnica de Catalunya, Laboratori d'Aplicacions Bioacústiques, Centre Tecnològic de Vilanova i la Geltrú, Avda. Rambla Exposició, s/n, Vilanova i la Geltrú, 08800 Spain.

${ }^{10}$ NCSR Demokritos, Institute of Nuclear and Particle Physics, Ag. Paraskevi Attikis, Athens, 15310 Greece.

${ }^{11}$ INFN, Sezione di Genova, Via Dodecaneso 33, Genova, 16146 Italy.

${ }^{12}$ University of Granada, Dept. of Computer Architecture and Technology/CITIC, 18071 Granada, Spain.

${ }^{13}$ Friedrich-Alexander-Universität Erlangen-Nürnberg, Erlangen Centre for Astroparticle Physics, Erwin-Rommel-Straße 1, 91058 Erlangen, Germany.

${ }^{14}$ Universitat Politècnica de València, Instituto de Investigación para la Gestión Integrada de las Zonas Costeras, C/ Paranimf, 1, Gandia, 46730 Spain.

${ }^{15}$ Nikhef, National Institute for Subatomic Physics, PO Box 41882, Amsterdam, 1009 DB Netherlands.

${ }^{16}$ University Mohammed V in Rabat, Faculty of Sciences, 4 av. Ibn Battouta, B.P. 1014, R.P. 10000 Rabat, Morocco.

${ }^{17}$ INFN, Sezione di Bologna, v.le C. Berti-Pichat, 6/2, Bologna, 40127 Italy.

\footnotetext{
${ }^{1}$ also at Dipartimento di Fisica, INFN Sezione di Padova and Università di Padova, I-35131, Padova, Italy
} 
${ }^{18}$ Università di Bologna, Dipartimento di Fisica e Astronomia, v.le C. Berti-Pichat, 6/2, Bologna, 40127 Italy.

${ }^{19}$ KVI-CART University of Groningen, Groningen, the Netherlands.

${ }^{20}$ INFN, Laboratori Nazionali del Sud, Via S. Sofia 62, Catania, 95123 Italy.

${ }^{21}$ North-West University, Centre for Space Research, Private Bag X6001, Potchefstroom, 2520 South Africa.

${ }^{22}$ Instituto Español de Oceanografía, Unidad Mixta IEO-UPV, C/ Paranimf, 1, Gandia, 46730 Spain.

${ }^{23}$ University Mohammed I, Faculty of Sciences, BV Mohammed VI, B.P. 717, R.P. 60000 Oujda, Morocco.

${ }^{24}$ Subatech, IMT Atlantique, IN2P3-CNRS, Université de Nantes, 4 rue Alfred Kastler - La Chantrerie, Nantes, BP 2072244307 France.

${ }^{25}$ Università di Salerno e INFN Gruppo Collegato di Salerno, Dipartimento di Matematica, Via Giovanni Paolo II 132, Fisciano, 84084 Italy.

${ }^{26}$ ISS, Atomistilor 409, Măgurele, RO-077125 Romania.

${ }^{27}$ University of Amsterdam, Institute of Physics/IHEF, PO Box 94216, Amsterdam, 1090 GE Netherlands.

${ }^{28}$ TNO, Technical Sciences, PO Box 155, Delft, 2600 AD Netherlands.

${ }^{29}$ Università degli Studi della Campania "Luigi Vanvitelli", Dipartimento di Matematica e Fisica, viale Lincoln 5, Caserta, 81100 Italy.

${ }^{30}$ Università La Sapienza, Dipartimento di Fisica, Piazzale Aldo Moro 2, Roma, 00185 Italy.

${ }^{31}$ Università di Bologna, Dipartimento di Ingegneria dell'Energia Elettrica e dell'Informazione "Guglielmo Marconi", Via dell'Università 50, Cesena, 47521 Italia.

${ }^{32}$ Cadi Ayyad University, Physics Department, Faculty of Science Semlalia, Av. My Abdellah, P.O.B. 2390, Marrakech, 40000 Morocco.

${ }^{33}$ University of the Witwatersrand, School of Physics, Private Bag 3, Johannesburg, Wits 2050 South Africa.

${ }^{34}$ Università di Catania, Dipartimento di Fisica e Astronomia "Ettore Majorana", Via Santa Sofia 64, Catania, 95123 Italy.

${ }^{35}$ INFN, LNF, Via Enrico Fermi, 40, Frascati, 00044 Italy.

${ }^{36}$ INFN, Sezione di Bari, via Orabona, 4, Bari, 70125 Italy.

${ }^{37}$ International Centre for Radio Astronomy Research, Curtin University, Bentley, WA 6102, Australia.

${ }^{38}$ University of Bari, Via Amendola 173, Bari, 70126 Italy.

${ }^{39}$ University Würzburg, Emil-Fischer-Straße 31, Würzburg, 97074 Germany.

${ }^{40}$ Western Sydney University, School of Computing, Engineering and Mathematics, Locked Bag 1797, Penrith, NSW 2751 Australia.

${ }^{41}$ Università di Salerno e INFN Gruppo Collegato di Salerno, Dipartimento di Fisica, Via Giovanni Paolo II 132, Fisciano, 84084 Italy.

${ }^{42}$ Università di Genova, Via Dodecaneso 33, Genova, 16146 Italy.

${ }^{43}$ Normandie Univ, ENSICAEN, UNICAEN, CNRS/IN2P3, LPC Caen, LPCCAEN, 6 boulevard Maréchal Juin, Caen, 14050 France.

${ }^{44}$ University of Granada, Dpto. de Física Teórica y del Cosmos \& C.A.F.P.E., 18071 Granada, Spain.

${ }^{45}$ NIOZ (Royal Netherlands Institute for Sea Research), PO Box 59, Den Burg, Texel, 1790 AB, the Netherlands.

${ }^{46}$ Tbilisi State University, Department of Physics, 3, Chavchavadze Ave., Tbilisi, 0179 Georgia.

${ }^{47}$ Leiden University, Leiden Institute of Physics, PO Box 9504, Leiden, 2300 RA Netherlands.

${ }^{48}$ National Centre for Nuclear Research, 02-093 Warsaw, Poland.

${ }^{49}$ Institut Universitaire de France, 1 rue Descartes, Paris, 75005 France.

${ }^{50}$ University of Johannesburg, Department Physics, PO Box 524, Auckland Park, 2006 South Africa.

${ }^{51}$ Eberhard Karls Universität Tübingen, Institut für Astronomie und Astrophysik, Sand 1, Tübingen, 72076 Germany.

${ }^{52}$ Università di Pisa, Dipartimento di Fisica, Largo Bruno Pontecorvo 3, Pisa, 56127 Italy.

${ }^{53}$ Laboratoire Univers et Particules de Montpellier, Place Eugène Bataillon - CC 72, Montpellier Cédex 05, 34095 France.

${ }^{54}$ Friedrich-Alexander-Universität Erlangen-Nürnberg, Remeis Sternwarte, Sternwartstraße 7, 96049 Bamberg, Germany.

${ }^{55}$ Université de Haute Alsace, 68100 Mulhouse Cedex, France.

${ }^{56}$ University of Münster, Institut für Kernphysik, Wilhelm-Klemm-Str. 9, Münster, 48149 Germany.

${ }^{57}$ Utrecht University, Department of Physics and Astronomy, PO Box 80000, Utrecht, 3508 TA Netherlands.

${ }^{58}$ INFN, CNAF, v.le C. Berti-Pichat, 6/2, Bologna, 40127 Italy. 